\title{
Smoking Withdrawal Modulates Right Inferior Frontal Cortex but not Presupplementary Motor Area Activation During Inhibitory Control
}

\author{
Rachel V Kozink', Scott H Kollins' and F Joseph McClernon*, I,2,3 \\ 'Department of Psychiatry and Behavioral Sciences, Duke University Medical Center, Durham, NC, USA; ${ }^{2}$ Brain Imaging and Analysis Center, \\ Duke University Medical Center, Durham, NC, USA; ${ }^{3}$ Durham Veterans Affairs Medical Center, and VISN 6 Mental Illness Research, Education, \\ and Clinical Center, Durham, NC, USA
}

\begin{abstract}
Smokers exhibit decrements in inhibitory control (IC) during withdrawal. The objective of this study was to investigate the neural basis of these effects in critical substrates of IC - right inferior frontal cortex (rIFC) and presupplementary motor area (pre-SMA). Smokers were scanned following smoking as usual and after 24-h smoking abstinence. During scanning they completed a Go/No-Go task that required inhibiting responses to infrequent STOP trials. Event-related brain activation in response to successfully inhibited STOP trials was evaluated in two regions of interest: $\mathrm{IIFC}(10 \mathrm{~mm}$ sphere, $x=40, y=30, z=26)$ and pre-SMA $(10 \mathrm{~mm}$ sphere, $x=2, y=18, z=40)$. Smoking abstinence robustly increased errors of commission on STOP trials ( 37.1 vs $24.8 \%$ in the satiated condition, $p<0.001$ ) while having no effects on GO trial accuracy or reaction time (RT). In rIFC, smoking abstinence was associated with a significantly increased event-related BOLD signal $(p=0.026)$. Pre-SMA was unaffected by smoking condition. The results of this preliminary study suggest that successful IC during withdrawal is associated with increased processing demands on a cortical center associated with attention to inhibitory signals.

Neuropsychopharmacology (2010) 35, 2600-2606; doi:I0.I038/npp.20I0.154; published online 22 September 20I0
\end{abstract}

Keywords: inhibitory control; smoking; $\mathrm{fMRl}$; withdrawal; inferior frontal cortex; pre-supplementary motor area

\section{INTRODUCTION}

An essential feature of drug addiction is deficient inhibitory control (IC), or the ability to withhold a prepotent or overlearned response. These deficits manifest themselves not only in an inability to control urges to use drugs (Kalivas and Volkow, 2005) but also in an inability to inhibit responses not directly related to drug use. Numerous studies, for instance, have demonstrated that addicted individuals perform worse than healthy controls on laboratory tasks requiring IC (Fillmore and Rush, 2002; Lane et al, 1998; Spinella, 2002).

Deficits in IC among drug abusers are thought to result from aberrant functioning of frontal lobe areas that control the balance between behavioral activation and inhibition (Koob and Volkow, 2009). Supportive neuroimaging studies have shown relative hypoactivation of frontal and cingulate

*Correspondence: Dr FJ McClernon, Duke University Medical Center, Box 2770I, Durham, NC 27708, USA, Tel: +9 196683987 ,

Fax: + 919668 5088, E-mail: mccle01 I@mc.duke.edu

Received 12 July 2010; revised 10 August 2010; accepted II August 2010 brain areas during response inhibition in cocaine users compared with non-users (Hester and Garavan, 2004; Kaufman et al, 2003; Li et al, 2008). Similarly, marijuana users exhibit differential patterns of right frontal and cingulate activation during response inhibition (Gruber and Yurgelun-Todd, 2005; Tapert et al, 2007). In addition, other cortical areas, including right insula (Kaufman et al, 2003), have been implicated in IC deficits.

Nicotine-dependent individuals, in addition to having baseline IC deficits as seen in other addicted populations (Spinella, 2002), exhibit further worsening of IC after tobacco cessation (Hatsukami et al, 1989; McClernon et al, 2008; Pettiford et al, 2007; Powell et al, 2002; Zack et al, 2001). In laboratory studies, abstinence from smoking has been shown to result in worse antisaccade performance (Pettiford et al, 2007; Powell et al, 2002) and more commission errors on continuous performance tasks (CPT) (Hatsukami et al, 1989; McClernon et al, 2008; Zack et al, 2001). Moreover, the administration of nicotine lozenges or gum to abstinent smokers results in better inhibitory control compared with placebo (Dawkins et al, 2007; Larrison et al, 2004), suggesting that the effects of smoking abstinence on response inhibition are due to abstinence 
from nicotine and not due to non-nicotine factors associated with smoking (eg, taste, behavioral ritual, and so on; also see Bekker et al, 2005). Finally, the degree to which nicotine (4 mg lozenge) improves antisaccade performance after overnight abstinence was found to positively correlate with self-reported nicotine dependence and also predicted relapse during the first week after quitting (Powell et al, 2004). As such, abstinence-induced IC deficits may be a critical mechanism underlying smoking lapse/relapse.

To our knowledge, no studies to date have investigated the neural substrates of IC deficits during smoking withdrawal. In this preliminary study, dependent smokers underwent functional MRI scans while they completed an ' $\mathrm{X}-\mathrm{Y}$ ' variant of the Go/No-Go task (Garavan et al, 2003; Garavan et al, 2002). On the basis of previous studies, we hypothesized that smoking abstinence would worsen IC (ie, increase errors of commission on stop trials). With respect to imaging data, we hypothesized that smoking abstinence would either (1) decrease activation in response to STOP signals reflecting a functional deficit in IC or (2) increase activation possibly reflecting compensatory activation. To minimize type I error, we restricted our imaging analyses to two regions of interest (ROIs) identified in a meta-analysis of Go/No-Go studies (Simmonds et al, 2008) and thought to be critical substrates of inhibitory control (Aron and Poldrack, 2005; Garavan et al, 2006): right inferior frontal cortext (rIFC) and presupplementary motor area (pre-SMA).

\section{MATERIALS AND METHODS}

\section{Participants}

A total of 18 adult dependent smokers (7 women) completed all aspects of the study. To be enrolled, participants had to report smoking $\geqslant 10$ cigarettes per day for at least 2 years, have an afternoon expired-air carbon monoxide (CO) level greater than 10 p.p.m. (to establish smoking status), be right handed, free of serious health problems (eg, hypertension), not currently undergoing treatment for a psychiatric illness, free of medications altering CNS functioning, test negative for illicit drug use, not have any conditions making MRI research unsafe, and, among females, have a negative serum pregnancy test. Two participants were excluded from analyses because they reported falling asleep in the scanner; one participant was excluded because of an incidental finding during acquisition of a structural scan. Thus, the final sample consisted of 15 dependent smokers.

\section{Procedure}

All participants first completed a 1.5 -h screening session, in which they read and signed an Institutional Review Boardapproved informed consent form, completed questionnaires regarding smoking history and suitability for fMRI research, and provided a breath sample. They were also placed in a mock scanner in order to habituate them to the scanning environment.

Participants who passed screening then completed a 1-h training session in which they were familiarized with the task. After training, participants completed two fMRI sessions: once while smoking satiated and once after a 24-h period of biologically verified smoking abstinence, which, as in previous studies, they were required to maintain for an additional $24 \mathrm{~h}$ after the scanning session (McClernon et al, 2009; Kozink et al, 2010). Requiring 24-h abstinence after the abstinence-condition scan minimizes any confounds that might stem from anticipating smoking. As part of the informed consent process, participants were informed that participation would require 48 -h abstinence and signed an honor statement promising to maintain abstinence for that period of time. Session order was counterbalanced. Continued abstinence was also verified $24 \mathrm{~h}$ after the abstinent-condition scanning session during a 0.5 -h quit check in which breath and saliva samples were collected.

\section{Biochemical Verification}

Expired-air $\mathrm{CO}$ concentrations were measured using a handheld CO monitor (Vitalograph, Lenexa, KS) and calculated by subtracting the background (ambient) $\mathrm{CO}$ from the peak $\mathrm{CO}$ reading. Criterion $\mathrm{CO}$ in the abstinence condition was $\leqslant 8$ p.p.m. Saliva samples were stored in an on-site $-80^{\circ} \mathrm{C}$ freezer. Assays for nicotine (collected at scanning sessions and 24-h quit check and used to characterize manipulation effects on nicotine levels) were performed using gas chromatography modified for use of a capillary column (Jacob et al, 1981).

Nicotine dependence was measured using the six-item Fagerström Test for Nicotine Dependence (FTND; Heatherton et al, 1991).

\section{fMRI Task}

Participants completed an $\mathrm{X}-\mathrm{Y}$ Go/NoGo task (Garavan et al, 2002). Briefly, participants were presented with a streaming, alternating pattern of the letters $\mathrm{X}$ and $\mathrm{Y}$ at $1 \mathrm{~Hz}$. They were instructed to press the button for each stimulus presented (GO trials), unless the alternating pattern was disrupted, at which point they were to withhold their response to the repeated letter (STOP trials). The interval between STOP trials was jittered between 10 and $24 \mathrm{~s}$ $($ mean $=16.5 \mathrm{~s})$. To balance performance across the sample, stimulus timing was individually tailored on the basis of a participant's performance at training. Stimulus onset asynchrony was held constant at $1 \mathrm{~s}$, but the duration of the stimulus was manipulated between $600,700,800$, and $900 \mathrm{~ms}$, followed by a blank screen of 400,300, 200, or $100 \mathrm{~ms}$, respectively. The appropriate timing was selected on the basis of the parameters that produced at least $50 \%$ accuracy on STOP trials, calculated as the number of correctly inhibited responses on STOP trials divided by the total number of STOP trials. Total task time was $12.5 \mathrm{~min}$ and included 708 GO stimuli and 42 STOP stimuli. Accuracy for GO and STOP trials and reaction time (RT) on GO trials were analyzed using paired $t$-tests in SPSS.

\section{Imaging Methods}

Scanning was preformed on a 4.0-T GE LX NVi scanner with 41-mT/m gradients (General Electric, Waukesha, Wisconsin). Blood oxygen level-dependent (BOLD) functional images 
were collected for 34 contiguous slices ( $4 \mathrm{~mm}$ thick) parallel to the horizontal plane connecting the anterior and posterior commissures. An inverse spiral pulse sequence sensitive to BOLD contrast was used, with $\mathrm{TR}=1.5 \mathrm{~s}, \mathrm{TE}=6 \mathrm{~ms}$, FOV $=24 \mathrm{~cm}$, matrix $=64 \times 64$, flip angle $=60^{\circ}$, and inplane resolution $=3.75 \mathrm{~mm}^{2}$. After completion of the functional data collection, a T1-weighted 3D fast spoiled gradient-recalled (FSPGR) structural image was collected for 68 slices $(1.9 \mathrm{~mm}$ thick $)$, with $\mathrm{TR}=12.3 \mathrm{~s}, \mathrm{TE}=5.4 \mathrm{~ms}$, FOV $=24 \mathrm{~cm}$, matrix $=256 \times 256$, flip angle $=20^{\circ}$, and inplane resolution $=0.9375 \mathrm{~mm}^{2}$.

\section{Data Analysis}

Preprocessing was conducted using statistical parametric mapping software (SPM5; Wellcome Department of Imaging Neuroscience, London) to remove noise and artifacts. The first four volumes of each run were discarded to allow for T1 stabilization. All functional images underwent correction for acquisition timing and for head motion using rigid-body rotation and translation (Friston et al, 1994). Each participant's data were then subsequently warped into a standard stereotaxic space (Montreal Neurological Institute) with an isotropic $2 \mathrm{~mm}$ voxel size and smoothed with an $8 \mathrm{~mm}$ FWHM Gaussian filter.

Each participant's data from each session were entered into a first-level whole brain analysis using the General Linear Model (Friston et al, 1994). Two event types were included in the model: correctly inhibited responses (STOPS) and errors of commission. Each event type was coded as a delta regressor and convolved with a canonical HRF. Motion parameters were also included in the model as a nuisance covariate. WFU Pickatlas (Maldjian et al, 2004; Maldjian et al, 2003) was used to create the two ROIs of interest (rIFC and pre-SMA) by drawing spheres with $10 \mathrm{~mm}$ radii centered around $x=40, y=30, z=26$ and $x=2, y=18, z=40$, respectively (Simmonds et al, 2008). Using MarsBar (Brett et al, 2002), percentage of BOLD signal change for the correctly identified STOP trials within each ROI was extracted for each participant and condition. The signal values from the peak of the hemodynamic response (HDR, $6 \mathrm{~s}$ after onset) were then input into SPSS 17.0 to examine differences in the amplitude of response between conditions using paired $t$-tests. Given the small number of tests conducted, all hypotheses were tested at $p<0.05$.

\section{RESULTS}

\section{Sample Characteristics}

The final sample $(n=15)$ was $40.0 \%$ female $(n=6)$. Reported racial/ethnic group membership was $86.7 \%$ Caucasian $(n=13)$ and $13.3 \%$ African American $(n=2)$. Mean age was 31.4 years $(S D=10.4)$. The sample reported smoking a mean of 16.2 cigarettes/day $(\mathrm{SD}=4.1)$ for 14.2 years $(\mathrm{SD}=9.4)$. The mean FTND score was $4.8(\mathrm{SD}=1.6)$, suggesting that the sample was moderately nicotine dependent. At screening, the mean expired-breath CO concentration was $24.7(\mathrm{SD}=13.6)$, whereas the mean salivary cotinine was $269.9 \mathrm{ng} / \mathrm{ml}(\mathrm{SD}=146.8)$.

\section{Abstinence Verification}

Expired-breath $\mathrm{CO}$ concentrations indicated compliance with study requirements. In the satiated condition, mean CO level (p.p.m.) was $29.2(\mathrm{SD}=20.3)$, whereas in the 24 -h abstinent condition, mean $\mathrm{CO}$ was $2.2(\mathrm{SD}=0.9)$. Mean $\mathrm{CO}$ $24 \mathrm{~h}$ after the abstinent fMRI session was $2.0(\mathrm{SD}=2.1)$.

The results of salivary nicotine analyses were consistent with $\mathrm{CO}$ results and also indicative of compliance. In the satiated and abstinent conditions, mean salivary nicotine $(\mathrm{ng} / \mathrm{ml}) \quad$ was $\quad 406.8 \quad(\mathrm{SD}=293.7)$ and $13.9 \quad(\mathrm{SD}=8.6)$, respectively.

\section{Behavioral Results}

Performance variables (GO trial accuracy and RT; STOP trial accuracy) were entered into paired $t$-tests evaluating the effects of condition (satiated, abstinent). Consistent with previous studies, abstinence was associated with significantly decreased accuracy on STOP trials $(t=-3.16$, $p=0.007 ;$ satiated mean $=75.2 \%, \quad \mathrm{SD}=13.1 ;$ abstinent mean $=62.9 \%, S D=22.1)$. Smoking abstinence significantly affected neither GO accuracy $(p=0.1$; satiated mean $=99.5$, $\mathrm{SD}=0.8$; abstinent mean $=98.8, \mathrm{SD}=1.8)$ nor $\mathrm{RT}(p=0.86$; satiated mean $=334.3, \mathrm{SD}=47.9$; abstinent mean $=332.8$, $\mathrm{SD}=63.0)$.

\section{fMRI Results}

We examined the effect of smoking condition on HDR amplitude in rIFC and pre-SMA ROIs. The amplitude of HDR at $6 \mathrm{~s}$ after stimulus onset in rIFC in response to STOP trials was greater in the abstinent as compared with the satiated condition $(t(14)=-2.84, p=0.026$, see Figure 1$)$, whereas smoking condition had no effect on pre-SMA activity.

\section{DISCUSSION}

This study used event-related fMRI to examine the effects of smoking abstinence on neural correlates of IC during a Go/No-Go task. Consistent with previous studies, smoking abstinence worsened IC as measured by errors of commission on STOP trials, while leaving GO trial performance unaffected. In addition, smoking abstinence resulted in increased IC activity in rIFC compared with satiety, but had no effect on pre-SMA. The findings provide novel information regarding the effects of smoking abstinence on neural activity associated with IC and suggest that greater neural resources are dedicated to IC during withdrawal in order to maintain performance.

\section{Effects of Smoking Abstinence on Inhibitory Control}

In the present study, smoking abstinence worsened IC as evidenced by more errors of commission on STOP trials of a Go/NoGo task. The size of this effect was large (Cohen's $d=0.93$ ), with $87 \%$ (13 of 15) participants exhibiting decreased IC. At the same time, neither RT nor accuracy on GO trials was affected by smoking abstinence. These findings replicate numerous previous studies that have observed abstinence-induced IC deficits across multiple 

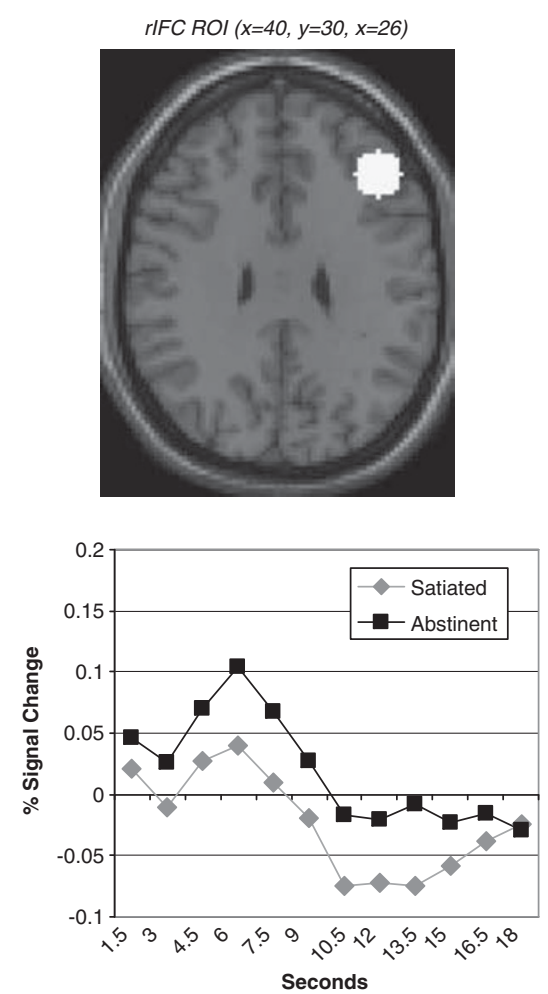
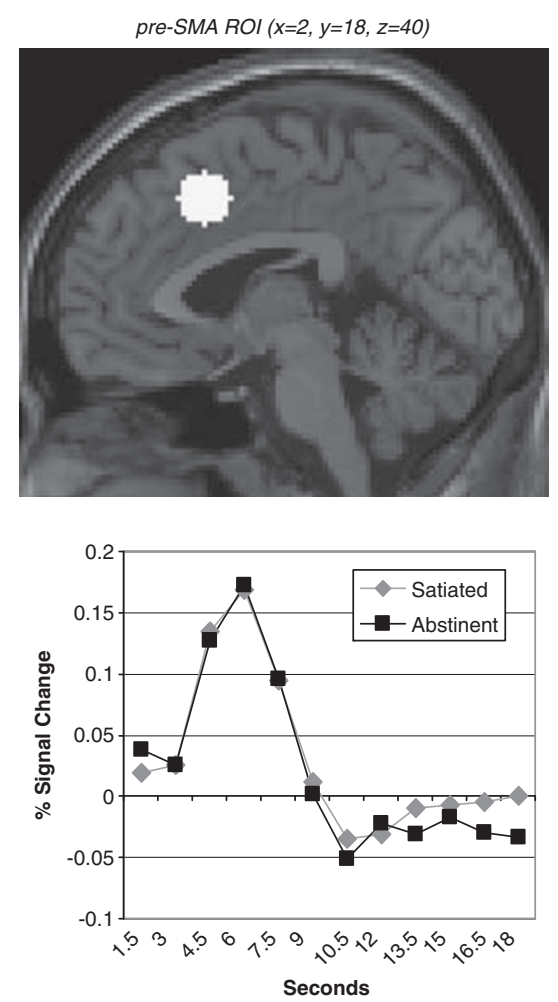

Figure I Percentage signal change in rIFC (left column) and pre-SMA (right column) regions of interests (ROIs) for STOP trials in the satiated and 24-h abstinent conditions. Top row, ROls were spheres with $10 \mathrm{~mm}$ radii centered around $x=40, y=30, z=26$ and $x=2, y=18, z=40$ (MNI) for rIFC and pre-SMA, respectively (Simmonds et al, 2008). Bottom row. Signal at the peak of the hemodynamic response was greater in the abstinent as compared with satiated condition, $p=0.026$ in rIFC; no difference was observed in pre-SMA.

tasks, including antisaccade and continuous performance tasks (Hatsukami et al, 1989; McClernon et al, 2008; Pettiford et al, 2007; Powell et al, 2002). For instance, we previously showed that overnight smoking abstinence decreased smokers' ability to withhold shifting their gaze to novel visual stimuli (antisaccade trials), but had no effect on prosaccade trial accuracy (Pettiford et al, 2007).

The imaging results of the present study provide novel evidence that IC deficits during withdrawal are associated with modulation of transient brain activation in rIFC but not in the medial frontal (pre-SMA) cortex. We specifically chose an ROI in right IFC as 1) activations in this region have been observed across numerous imaging studies of IC (Garavan et al, 2006; Garavan et al, 1999; Konishi et al, 1999) and 2) lesions of this region result in IC deficits (Aron et al, 2003; Aron et al, 2004). Pre-SMA has similarly been implicated in inhibitory control (Aron et al, 2007; Floden and Stuss, 2006; Isoda and Hikosaka, 2007; Mostofsky et al, 2003). Activation in these areas is common to inhibition across response modalities (Xue et al, 2008). In a recent meta-analysis of Go/No-Go fMRI studies (Simmonds et al, 2008), successful inhibition most robustly activated regions in (1) the right frontal cortex on the border between IFG and MFG and (2) pre-SMA. The coordinates of these activations were used as the centers of the ROI used in our analysis.

The exact nature of the role of rIFC in IC remains unclear. The rIFC is part of the ventral attention system involved in the orienting of attention to behaviorally relevant or salient stimuli (Corbetta et al, 2008; Hampshire et al, 2009; Linden et al, 1999), and recent imaging studies suggest that its role in IC is more related to these attentional functions than to the cessation of behavior per se (Chao et al, 2009; Duann et al, 2009; Hampshire et al, 2010; Sharp et al, 2010). For instance, a recent fMRI study in which attentional and behavioral control aspects of IC were dissociated showed that $\mathrm{rIFC}$ is not involved in IC after removing activation associated with attentional capture (Sharp et al, 2010). In this same study, activation in pre-SMA survived any correction for attentional processes. In addition, a more refined view of rIFC is emerging, in which specific subregions are differentially involved in aspects of inhibitory control, including selective attention to task-relevant stimuli and updating of behavioral plans (Chikazoe et al, 2009; Verbruggen et al, 2010). In an fMRI study using a Go/ No-Go task that included infrequent GO in addition to infrequent STOP trials (Chikazoe et al, 2009), the posterior aspect of the inferior frontal gyrus (pIFG) is active in response to STOP trials after removing activation in response to infrequent GO trials (ie, response inhibition specific), whereas activation is observed in inferior frontal junction (IFJ) by infrequent as compared with frequent GO trials (ie, orienting of attention novel/salient stimuli). Similarly, an rTMS study (Verbruggen et al, 2010) that selectively inactivated right IFC subregions found that rIFG is critical to inhibitory control through its updating of action plans, whereas IFJ is involved in attention to task-critical stimuli. As such, the accumulating literature 
suggests that successful IC is dependent on right frontalmediated attentional control and action planning/updating, and medial prefrontal (pre-SMA)-mediated behavioral control (ie, cessation of a prepotent response).

Our analysis was restricted to trials in which participants successfully inhibited a response. As such, the finding that smoking abstinence increased transient activation in rIFC suggests the possibility that abstinence places increased attentional demands in order to successfully inhibit behavior even at suboptimal levels. Other studies have observed similar effects of smoking abstinence to increase (or nicotine to decrease) transient activation during attention (Azizian et al, 2010; Kozink et al, 2010; Thiel and Fink, 2008; Vossel et al, 2008). In addition, consistent with the interpretation that smoking abstinence increases demands on IFC processing to maintain performance, drug users, compared with non-drug-using controls, exhibit increased frontal activation during successful inhibition, even when no differences in performance are observed (Roberts and Garavan, 2010; Tapert et al, 2007).

Although our findings suggest that smoking abstinence modulates rIFC function, our findings do not rule out the possibility that smoking abstinence alters the operation of behavioral control centers including pre-SMA, as we only examined trials in which behavioral control was successful. However, the fact that smoking abstinence did not increase activation in pre-SMA suggests the possibility that no additional demands are placed on this region in the withholding of responses. As such, our findings provide preliminary evidence that abstinence-induced inhibitory control deficits may involve deficits of attention to a greater degree than motor inhibition. This interpretation is consistent with a large literature showing that smoking abstinence robustly disrupts attentional processes (Heishman et al, 1994; Levin et al, 2006), including the detection of novel stimuli (Froeliger et al, 2009).

\section{Limitation and Future Directions}

Strengths of this study include the within-subjects design, use of a well-established task with known neural correlates, and an ROI-based analytical approach that reduces risk of type I error. However, several limitations should be noted. First, the sample size is small and, as such, the results should be considered preliminary. Moreover, the lack of a non-smoking control group complicates interpretation-greater activation in the abstinent condition may reflect either a return to normal presmoking levels or a disruption of baseline functioning. Assessments at time points more distal to abstinence would also help to address this question. In addition, relative to GO stimuli, STOP signals in the current paradigm are rare and thus responses to them may reflect inhibitory control, the detection of a novel stimulus, or some combination of these factors. Future studies that incorporate paradigms with tasks that differentiate neural activity associated with these two processes can help elucidate this question. On a related note, we used a single rIFC ROI based on previous research using the $\mathrm{X}-\mathrm{Y}$ Go/No-Go paradigm. However, as noted above, there is important functional variation in IFC subregions - future studies can make use of multiple IFC ROIs to further pinpoint smoking abstinence effects.
Finally, previous research has examined brain activation in response to errors of commission on STOP trials; however, adequate numbers of such trials were not available for analysis in the present study. Future studies in which task difficulty is adaptively modified to increase errors of commission can better address this issue.

\section{CONCLUSIONS}

This study is a novel investigation of the neural substrates that underlie disruption of IC during withdrawal and adds to the growing database on the effects of smoking abstinence on neurocognitive functioning (McClernon and Gilbert, 2004). We observed that smoking abstinence increased activation in rIFC, which suggests that IC deficits following abstinence are due to increased demand on attention processes rather than the disruption or modulation of stopping processes per se. Future studies using tasks that allow for the dissociation of attentional and behavioral control aspects of IC can shed additional light on the mechanisms that underlie abstinence-induced IC deficits. In addition, the present results suggest that rIFC activation may be a clinically relevant biomarker of smoking cessation treatment effects and a potential target for new interventions.

\section{ACKNOWLEDGEMENTS}

We thank Avery Lutz, Natalie Goutkin, and Luke Pool for their assistance with data acquisition. This research was supported by NIDA Grant K23DA017261 to FJM, NIDA Grant K24DA023464 to SHK, and by an unrestricted research grant from Philip Morris USA (Dr Jed E. Rose, PI).

\section{DISCLOSURE}

Ms Kozink reports no conflict of interest. Dr Kollins has received research funding and/or consulting fees from Shire Pharmaceuticals, Otsuka Pharmaceuticals, Supernus Pharmaceuticals, and from the National Institute on Drug Abuse. Dr McClernon reports having research funding from the National Institute on Drug Abuse and the Atkins Foundation; and salary support from an unrestricted grant from Philip Morris USA (Dr Jed E. Rose, PI).

\section{REFERENCES}

Aron AR, Behrens TE, Smith S, Frank MJ, Poldrack RA (2007). Triangulating a cognitive control network using diffusionweighted magnetic resonance imaging (MRI) and functional MRI. J Neurosci 27: 3743-3752.

Aron AR, Fletcher PC, Bullmore ET, Sahakian BJ, Robbins TW (2003). Stop-signal inhibition disrupted by damage to right inferior frontal gyrus in humans. Nat Neurosci 6: 115-116.

Aron AR, Poldrack RA (2005). The cognitive neuroscience of response inhibition: relevance for genetic research in attentiondeficit/hyperactivity disorder. Biol Psychiatry 57: 1285-1292.

Aron AR, Robbins TW, Poldrack RA (2004). Inhibition and the right inferior frontal cortex. Trends Cogn Sci 8: 170-177.

Azizian A, Nestor LJ, Payer D, Monterosso JR, Brody AL, London ED (2010). Smoking reduces conflict-related anterior cingulate 
activity in abstinent cigarette smokers performing a stroop task. Neuropsychopharmacology 35: 775-782.

Bekker EM, Bocker KB, Van Hunsel F, van den Berg MC, Kenemans JL (2005). Acute effects of nicotine on attention and response inhibition. Pharmacol Biochem Behav 82: 539-548.

Brett M, Anton J-L, Valabregue R, Poline J-B (2002). Region of interest analysis using an SPM toolbox. In 8th International Conference on Functional Mapping of the Human Brain. Sendai: Japan.

Chao HH, Luo X, Chang JL, Li CS (2009). Activation of the presupplementary motor area but not inferior prefrontal cortex in association with short stop signal reaction time-an intrasubject analysis. BMC Neurosci 10: 75.

Chikazoe J, Jimura K, Asari T, Yamashita K, Morimoto H, Hirose $S$, et al (2009). Functional dissociation in right inferior frontal cortex during performance of go/no-go task. Cereb Cortex 19: $146-152$.

Corbetta M, Patel G, Shulman GL (2008). The reorienting system of the human brain: from environment to theory of mind. Neuron 58: 306-324.

Dawkins L, Powell JH, West R, Powell J, Pickering A (2007). A double-blind placebo-controlled experimental study of nicotine: II-Effects on response inhibition and executive functioning. Psychopharmacology (Berl) 190: 457-467.

Duann JR, Ide JS, Luo X, Li CS (2009). Functional connectivity delineates distinct roles of the inferior frontal cortex and presupplementary motor area in stop signal inhibition. J Neurosci 29: 10171-10179.

Fillmore MT, Rush CR (2002). Impaired inhibitory control of behavior in chronic cocaine users. Drug Alcohol Depend 66: 265-273.

Floden D, Stuss DT (2006). Inhibitory control is slowed in patients with right superior medial frontal damage. J Cogn Neurosci 18: $1843-1849$.

Friston KJ, Jezzard P, Turner R (1994). Analysis of functional MRI time-series. Human Brain Mapping 1: 153-171.

Froeliger B, Gilbert DG, McClernon FJ (2009). Effects of nicotine on novelty detection and memory recognition performance: double-blind, placebo-controlled studies of smokers and nonsmokers. Psychopharmacology (Berl) 205: 625-633.

Garavan H, Hester R, Murphy K, Fassbender C, Kelly C (2006). Individual differences in the functional neuroanatomy of inhibitory control. Brain Res 1105: 130-142.

Garavan H, Ross TJ, Kaufman J, Stein EA (2003). A midline dissociation between error-processing and response-conflict monitoring. Neuroimage 20: 1132-1139.

Garavan H, Ross TJ, Murphy K, Roche RA, Stein EA (2002). Dissociable executive functions in the dynamic control of behavior: inhibition, error detection, and correction. Neuroimage 17: 1820-1829.

Garavan H, Ross TJ, Stein EA (1999). Right hemispheric dominance of inhibitory control: an event-related functional MRI study. Proc Natl Acad Sci USA 96: 8301-8306.

Gruber SA, Yurgelun-Todd DA (2005). Neuroimaging of marijuana smokers during inhibitory processing: a pilot investigation. Brain Res Cogn Brain Res 23: 107-118.

Hampshire A, Chamberlain SR, Monti MM, Duncan J, Owen AM (2010). The role of the right inferior frontal gyrus: inhibition and attentional control. Neuroimage 50: 1313-1319.

Hampshire A, Thompson R, Duncan J, Owen AM (2009). Selective tuning of the right inferior frontal gyrus during target detection. Cogn Affect Behav Neurosci 9: 103-112.

Hatsukami D, Fletcher L, Morgan S, Keenan R, Amble P (1989). The effects of varying cigarette deprivation duration on cognitive and performance tasks. J Subst Abuse 1: 407-416.

Heatherton TF, Kozlowski LT, Frecker RC, Fagerstrom KO (1991). The fagerstrom test for nicotine dependence: a revision of the fagerstrom tolerance questionnaire. $\mathrm{Br} J$ Addict 86: 1119-1127.
Heishman SJ, Taylor RC, Henningfield JE (1994). Nicotine and smoking: a review of effects on human performance. Experimental \& Clinical Psychopharmacology 2: 345.

Hester R, Garavan H (2004). Executive dysfunction in cocaine addiction: evidence for discordant frontal, cingulate, and cerebellar activity. J Neurosci 24: 11017-11022.

Isoda M, Hikosaka O (2007). Switching from automatic to controlled action by monkey medial frontal cortex. Nat Neurosci 10: 240-248.

Jacob III P, Wilson M, Benowitz NL (1981). Improved gas chromatographic method for the determination of nicotine and cotinine in biologic fluids. J Chromatogr 222: 61-70.

Kalivas PW, Volkow ND (2005). The neural basis of addiction: a pathology of motivation and choice. Am J Psychiatry 162: 1403-1413.

Kaufman JN, Ross TJ, Stein EA, Garavan H (2003). Cingulate hypoactivity in cocaine users during a GO-NOGO task as revealed by event-related functional magnetic resonance imaging. J Neurosci 23: 7839-7843.

Konishi S, Nakajima K, Uchida I, Kikyo H, Kameyama M, Miyashita Y (1999). Common inhibitory mechanism in human inferior prefrontal cortex revealed by event-related functional MRI. Brain 122( Part 5): 981-991.

Koob GF, Volkow ND (2009). Neurocircuitry of addiction. Neuropsychopharmacology 35: 217-238.

Kozink RV, Lutz AM, Rose JE, Froeliger B, McClernon FJ (2010). Smoking withdrawal shifts the spatiotemporal dynamics of neurocognition. Addiction Biology (in press).

Lane SD, Cherek DR, Dougherty DM, Moeller FG (1998). Laboratory measurement of adaptive behavior change in humans with a history of substance dependence. Drug Alcohol Depend 51: 239-252.

Larrison AL, Briand KA, Sereno AB (2004). Nicotine improves antisaccade task performance without affecting prosaccades. Hum Psychopharmacol 19: 409-419.

Levin ED, McClernon FJ, Rezvani AH (2006). Nicotinic effects on cognitive function: behavioral characterization, pharmacological specification, and anatomic localization. Psychopharmacology (Berl) 184: 523-539.

Li CS, Huang C, Yan P, Bhagwagar Z, Milivojevic V, Sinha R (2008). Neural correlates of impulse control during stop signal inhibition in cocaine-dependent men. Neuropsychopharmacology 33: 1798-1806.

Linden DE, Prvulovic D, Formisano E, Vollinger M, Zanella FE, Goebel R et al (1999). The functional neuroanatomy of target detection: an fMRI study of visual and auditory oddball tasks. Cereb Cortex 9: 815-823.

Maldjian JA, Laurienti PJ, Burdette JH (2004). Precentral gyrus discrepancy in electronic versions of the Talairach atlas. NeuroImage 21: 450.

Maldjian JA, Laurienti PJ, Kraft RA, Burdette JH (2003). An automated method for neuroanatomic and cytoarchitectonic atlas-based interrogation of fMRI data sets. NeuroImage 19: 1233.

McClernon FJ, Gilbert DG (2004). Human functional neuroimaging in nicotine and tobacco research: basics, background, and beyond. Nicotine Tob Res 6: 941-959.

McClernon FJ, Kollins SH, Lutz AM, Fitzgerald DP, Murray DW, Redman C et al (2008). Effects of smoking abstinence on adult smokers with and without attention deficit hyperactivity disorder: results of a preliminary study. Psychopharmacology (Berl) 197: 95-105.

McClernon FJ, Kozink RV, Lutz AM, Rose JE (2009). 24-h smoking abstinence potentiates fMRI-BOLD activation to smoking cues in cerebral cortex and dorsal striatum. Psychopharmacology (Berl) 204: 25-35.

Mostofsky SH, Schafer JG, Abrams MT, Goldberg MC, Flower AA, Boyce A et al (2003). fMRI evidence that the neural basis of response inhibition is task-dependent. Brain Res Cogn Brain Res 17: 419-430. 
Smoking fMRI of inhibitory control

RV Kozink et al

2606

Pettiford J, Kozink RV, Lutz AM, Kollins SH, Rose JE, McClernon FJ (2007). Increases in impulsivity following smoking abstinence are related to baseline nicotine intake and boredom susceptibility. Addict Behav 32: 2351-2357.

Powell J, Dawkins L, Davis RE (2002). Smoking, reward responsiveness, and response inhibition: tests of an incentive motivetional model. Biol Psychiatry 51: 151-163.

Powell JH, Pickering AD, Dawkins L, West R, Powell JF (2004). Cognitive and psychological correlates of smoking abstinence, and predictors of successful cessation. Addict Behave 29: 1407-1426.

Roberts GM, Garavan H (2010). Evidence of increased activation underlying cognitive control in ecstasy and cannabis users. Neuroimage 52: 429-435.

Sharp DJ, Bonnelle V, De Boissezon X, Beckmann CF, James SG, Patel MC et al (2010). Distinct frontal systems for response inhibition, attentional capture, and error processing. Proc Natl Aced Sci USA 107: 6106-6111.

Simmonds DJ, Pekan JJ, Mostofsky SH (2008). Meta-analysis of Go/ No-go tasks demonstrating that $\mathrm{PMRI}$ activation associated with response inhibition is task-dependent. Neuropsychologia 46: 224-232.
Spinella M (2002). Correlations between orbitofrontal dysfunction and tobacco smoking. Addict Biol 7: 381-384.

Tapers SF, Schweinsburg AD, Drummond SP, Paulus MP, Brown SA, Yang TT et al (2007). Functional MRI of inhibitory processing in abstinent adolescent marijuana users. Psychopharmacology (Bert) 194: 173-183.

Thiel CM, Fink GR (2008). Effects of the cholinergic agonist nicotine on reorienting of visual spatial attention and top-down attentional control. Neuroscience 152: 381-390.

Verbruggen F, Aron AR, Stevens MA, Chambers CD (2010). Theta burst stimulation dissociates attention and action updating in human inferior frontal cortex. Proc Natl Acad Sci USA 107: 13966-13971.

Vossel S, Thiel CM, Fink GR (2008). Behavioral and neural effects of nicotine on visuospatial attentional reorienting in nonsmoking subjects. Neuropsychopharmacology 33: 731-738.

Xe G, Aron AR, Poldrack RA (2008). Common neural substrates for inhibition of spoken and manual responses. Cere Cortex 18: 1923-1932.

Rack M, Belsito L, Scher R, Eissenberg T, Corrigall WA (2001). Effects of abstinence and smoking on information processing in adolescent smokers. Psychopharmacology (Bert) 153: 249-257.

Neuropsychopharmacology 